\title{
Figures filmiques d'ouvrières : travail, genre et dignité, variations sur une trilogie classique (1962-2011)
}

Work, Gender and Dignity. Variation on a Classic Triology: Women Workers in Women's Movies (1962-2011)

\section{Nicolas hatzfeld}

\section{OpenEdition}

\section{Journals}

Édition électronique

URL : http://journals.openedition.org/clio/11585

DOI : 10.4000/clio.11585

ISSN : $1777-5299$

Éditeur

Belin

Édition imprimée

Date de publication : 31 décembre 2013

Pagination : 79-96

ISSN : $1252-7017$

Référence électronique

Nicolas hatzfeld, «Figures filmiques d'ouvrières : travail, genre et dignité, variations sur une trilogie classique (1962-2011) », Clio. Femmes, Genre, Histoire [En ligne], 38 | 2013, mis en ligne le 31 décembre 2015, consulté le 19 avril 2019. URL : http://journals.openedition.org/clio/11585; DOI : 10.4000/ clio. 11585 


\section{Figures filmiques d'ouvrières : travail, genre et dignité, variations sur une trilogie classique (1962-2011)}

Nicolas HATZFELD

S’il est souvent admis que, depuis quelques décennies, les ouvriers sont frappés d'invisibilité dans la société française bien au-delà de la diminution effective de leur nombre réel ${ }^{1}$, que dire des ouvrières? Faut-il à leur sujet paraphraser la formule appliquée par le MLF à la femme du soldat inconnu, devant l'arc de Triomphe le 26 août 1970 ? Pour s'en faire une idée plus nette et contribuer au dessin de leur rôle dans l'histoire sociale récente, l'examen des représentations qui en sont établies fait ressortir un champ de références au sein duquel les figures d'ouvrières sont sans cesse recomposées. Si elle ne se contente pas d'évoquer leur simple fonction illustrative, l'analyse de ces portraits permet de mettre en question les rapports qu'entretiennent les situations effectives et leurs représentations. Les concordances qu'on trouve entre ces deux domaines, et plus encore les discordances, font ressortir les images attribuées aux ouvrières au sein de la société.

Parmi ces représentations, le cinéma offre un matériau d'une force expressive singulière, d'autant plus significative qu'elle repose sur la mobilisation de ressources généralement considérables et multiples. Chaque film est un processus global de mise en image passant par la conception, les négociations menant à sa mise en chantier, la mobilisation de métiers, de savoirs et de sensibilités aux différentes phases de sa réalisation, sa distribution et sa réception ${ }^{2}$. Certaines recherches ont montré la fécondité d'une analyse attentive à ce

\footnotetext{
Beaud \& Pialoux 1999; Vigna 2012.
}

2 Rot \& de Verdalle (dir.) 2013. 
parcours $^{3}$. Cependant l'étude présentée ici entend s'en tenir pour l'essentiel à la surface des films, en se contentant d'effectuer ici et là quelques remarques sur leur élaboration et leur trajectoire. L'objectif est plutôt d'explorer, parmi les productions destinées à une diffusion publique, un premier corpus réuni par le fait d'offrir une représentation d'ouvrières, que d'autres recherches pourront corriger et enrichir.

D'emblée, un tel ensemble s'avère particulièrement composite, mêlant notamment documentaires et fictions. La distinction entre ces deux genres, sans perdre sa pertinence, tend à accentuer des différences qui s'avèrent plus nuancées lorsqu'on précise l'examen. Certes, l'ancrage des premiers dans des situations réelles renforce leur statut de sources pour l'histoire sociale. Toutefois, les options prises au fil de la réalisation par de nombreux cinéastes combinent différents choix tels que les négociations préliminaires, le recours à des acteurs ou aux personnes directement concernées, l'authenticité des lieux ou leur reconstitution, la captation ou le jeu des scènes filmées, l'origine des sons, des voix et des discours, etc. L'ensemble de ces options filmiques réduit le clivage classique entre documentaires et fictions et peut conduire à privilégier l'unicité de l'ensemble, comme on le fera ici. Une autre typologie, correspondant aux destinations ultérieures des films, offrirait des pistes fécondes d'interprétation. L'exploitation dans des salles et, le cas échéant, son ampleur, la diffusion sur des chaines de télévision, la distribution par des réseaux cinéphiles ou militants ou, comme c'est aussi le cas, l'absence quasi totale de projection sont des aspects significatifs qui ne sont guère abordés, non plus que la distinction entre les intentions des réalisateurs et les carrières effectives des films. L'indistinction aide en retour à repérer la façon dont certains thèmes peuvent circuler à travers les types de film et se répondre suivant d'autres facteurs comme l'époque, le pays, la branche d'activité, etc. Elle incite à souligner les deux grandes relations à l'intersection desquelles se situe la condition des ouvrières : les rapports au travail et les relations de genre ${ }^{4}$. Ces deux grands domaines sur lesquels s'attardent de différentes façons les films rassemblés ici soulèvent des questions sur

\footnotetext{
3 Lindeperg 2007.

4 Gallot 2012.
} 
les types d'ouvrières représentées, leurs activités et leurs luttes ainsi que sur les agencements de leurs identités.

\section{Films de femmes, de France et d'ailleurs}

Pour envisager ces différents horizons et néanmoins tenir un certain cap dans la sélection, la recension s'appuie principalement sur l'imposant travail de prospection effectué depuis 35 ans par les organisatrices du Festival International de Films de Femmes de Créteil, qui offre une ouverture, une curiosité, une sensibilité précieuses ${ }^{5}$. Autant de traits, plus que de biais, qu'il convient d'avoir à l'esprit dans les interprétations d'ensemble. Le cœur de l'ensemble est donc constitué par les sélections effectuées depuis le début par cette équipe. D'autres titres sont issus d'une recherche de groupe ${ }^{6}$, de l'ouvrage de Michel Cadé ${ }^{7}$ ou des fonds de l'association Génériques ${ }^{8}$, enfin tirés de quêtes personnelles. L'ensemble constitué compte donc essentiellement des films réalisés par des cinéastes féminines sur des ouvrières. Le critère met l'accent sur l'émergence de la réalisation au féminin. Celle-ci, sous réserve de trouvailles à venir, apparaît sur ce sujet dans les années 1960, de façon encore marginale, et prend de la consistance après 1968, même si une partie des métiers de l'industrie du cinéma était féminisée depuis longtemps. Le choix n'est toutefois pas exclusif et certains films réalisés par des hommes, tels que Norma Rae, Coup pour coup ou, plus récemment, Rêves d'usine sont intégrés à l'ensemble. Même très lacunaire, cet ajout invite à s'interroger sur

5 Le Festival International de Films de Femmes de Créteil se tient chaque printemps depuis 1979 à la Maison des Arts de Créteil. Unique au monde, il fait connaître et soutient des films et des réalisatrices de tous les pays. Il entend promouvoir le regard des femmes sur les sociétés et mettre en valeur leurs différentes cultures. Depuis quelques années, ce Festival développe un Centre de ressources, IRIS, qui classe et numérise les archives des manifestations annuelles. Ce centre offre à l'étude toute l'année plus de 6000 films et de nombreux documents sur l'histoire du cinéma et des femmes. Un catalogue thématique existe à la consultation.

6 Groupe Nigwal. Hatzfeld, Rot \& Michel 2006.

7 Cadé 2004.

8 Je remercie ici Tangui Perron. 
l'éventuelle spécificité de genre de ces films, sous forme de pistes esquissées ici ou là en attente d'une comparaison plus systématique. Par contre, les films montrant des épouses et compagnes d'ouvriers ne sont pas retenus, même lorsqu'ils sont réalisés par des femmes, comme dans l'important documentaire Harlan County US A, réalisé par Barbara Kopple (1976). Un autre choix consiste à ne pas en rester au strict périmètre français, afin d'esquisser des comparaisons avec d'autres pays d'Europe, d'Amérique ou d'Asie même si, là encore, on est loin de viser un ensemble systématique.

Au fil des cinq décennies considérées, le statut des films évolue. Les deux premiers repérés, dans les années 1960, sont portés par des institutions, l'administration du cinéma d'État tchécoslovaque en voie de libéralisation et le parti communiste italien. Quelques années après 1968, surgit un groupe de films qui contourne peu ou prou l'industrie du cinéma, ou qui s'y oppose. Leurs auteures s'approprient souvent les nouvelles techniques de tournage, les caméras $16 \mathrm{~mm}$ à prise de son synchrone, puis les caméras vidéo, techniques économes et n'exigeant que des équipes minimales. Facilitant les rapports de proximité entre filmeurs et filmés, ces techniques correspondent aux démarches critiques, contestataires ou militantes d'une partie d'entre elles. Hollywood, toutefois, ne dédaigne pas cette veine en récompensant en 1976 le documentaire de Barbara Kopple puis, en 1979, la fiction Norma Rae. La décennie 1980 voit s’installer en France un grand silence tandis que, de-ci de-là, dans le monde, paraissent des œuvres qui prolongent et diversifient les questions laissées par la dynamique contestataire. Les années 2000 marquent une relance des productions françaises, notamment documentaires, tandis que s'enrichit le patrimoine venu d'ailleurs. La diversification des modes de diffusion favorise cette attention renouvelée.

Il ressort de l'ensemble une ouverture sur différents films d'Europe de l'Ouest et de l'Est ainsi que d'Amérique du Nord, à partir des années 1960-1970. Cet ensemble partage certaines évolutions des films français, tout au moins jusqu'aux années 2000 où l'inquiétude obsédante face aux fermetures d'usines distingue les œuvres d'Europe de l'Ouest. D'autres films venus des pays d'industrialisation vivace en Asie du Sud-Est (Indonésie, Corée, Vietnam) offrent pour cette dernière décennie un net contrepoint. 


\section{Des ouvrages et des jours}

Quelques films relèvent l'initiative de femmes qui, seules ou en groupe, conquièrent leur place sur des métiers traditionnellement masculins : une poseuse de cloisons d'origine finlandaise au Canada (Laila), un groupe féministe créant une entreprise de charpente en Californie (Raising the roof), une conductrice de camion dans le Kerala (Manjuben, truck driver) ou une ouvrière agricole taillant les vignes bordelaises (Femmes précaires). Mais de manière générale, les branches d'activité évoquées présentent des similitudes. Les images d'usines d'automobiles montrent en général une présence relativement banale d'ouvrières dans des ateliers mixtes où elles restent nettement minoritaires, travaillant en chaîne ou « donnant à manger aux robots » (Cadences en chaîne). On trouve la même discrétion minoritaire chez Michelin (Paroles de Bibs), au sein d'une imprimerie (Elefantenhaut) et à l'usine chimique marseillaise de Legre-Mante où, dans des ateliers venus du fond des âges le personnel produisait de l'acide tartrique. Les risques chimiques encourus reflètent une insalubrité qu'un autre film, In futura costruzione mentionne à propos de nombreux ouvriers morts de l'amiante dans une ancienne cimenterie.

La plupart des films, cependant, propose des ouvrières dans des activités où elles sont souvent majoritaires, parfois presque les seules salariées visibles. Cela apparaît dans les ateliers de câblage ou de garniture d'usines automobiles (Humain, trop bumain). C'est aussi le cas dans l'usine Samsonite (Liquidation totale) et surtout dans trois secteurs classiques de l'industrie au féminin. Le premier regroupe l'assemblage de précision dans l'électroménager, l'horlogerie et l'électronique (Essere donne; Lip; Grandin; Calor, une usine en perspective; Mon travail c'est capital; Sauf la lutte). Le textile est le plus largement représenté, tant en tissage qu'en confection, dans tous les continents et à toutes les époques. Les activités varient du tissage à la main de tapisseries artisanales (Dangter of the sun) à des couturières philippines «aptes à travailler sous pression » (Ouvrières du monde), selon une offre d'emploi, ou à d'autres qu'en Bretagne, un directeur se plaît à faire craquer devant la caméra (Maryflo). Enfin, les industries alimentaires montrent ici, sans fard, le visage le plus rude : les conserveries de fruits et de légumes (Sassedkata), et surtout l'abattage et la découpe massive de 
volaille en Russie (With love, Lily) et de poissons (En avoir (ou pas); Les filles de la sardine). Les jeunes filles de Tanger ne s'y trompent pas, partagées entre le groupe des «textiles » plutôt chanceux et celui des « crevettes» (Sur la planche), les plus mal loties.

Entre ces activités, les conditions de travail diffèrent, mais les films soulignent particulièrement leur pénibilité. Les conserveries animales sont marquées par le froid des ateliers et le contact lancinant avec les chairs découpées dont l'odeur semble coller à la peau (Sur la planche). Le bruit est plus répandu (Grandin; Elefantenhaut; We are not defeated), et devient particulièrement éprouvant dans les ateliers de tissage. Ne pouvant se faire entendre, c'est par inscription sur un carton qu'une ouvrière appelle ses voisins à la solidarité (Norma Rae). Parmi les traits communs de ces usines, on trouve les bas salaires déclinés dans tous les continents et à toutes les époques: dans l'Autriche de 1918 (Marianne. Ein recht für Alle), les États-Unis des années 1930 (Union Maids), la Russie postsoviétique, la Corée industrielle (We are not defeated), l'Angleterre d'avant la loi de 1970 sur l'égalité des salaires (We want sex equality), le Portugal de 1974 (Nous, ouvrières de la Sogantal) et l'actuelle Asie du Sud-Est (Rêves d'ouvrières, Ouvrières du monde). La France ne fait pas exception, même si des monteuses en vêtements regrettent que leurs consœurs philippines travaillent pour " un bol de riz » et que celles-ci, voyant sur un écran vidéo les maisons, les meubles et les voitures des premières, concluent sobrement : "Il y a une grande différence ». Ce bas niveau des rémunérations s'appuie généralement sur l'absence de qualification reconnue :

Chez Siemens, selon les termes du contrat, on est 2000 à devoir passer en $3^{e}$ catégorie. Mais jusqu'ici, seulement 150 y sont passées. C'est vraiment une honte. (Essere donne)

On trouve aussi la répétition de tâches brèves effectuées à haute cadence. Quand l'organisation d'un atelier de confection (Coup pour coup) ou d'une fabrique italienne de pâtes est encore rudimentaire, la contrainte de la "caporale », qui «nous crie tout le temps dessus », suffit à gâcher la vie. La conserverie de Tanger présente une surveillance de même nature quarante ans plus tard, avec une contremaîtresse passant dans les allées dans le dos des 
décortiqueuses. Les systèmes de salaire au rendement sont un autre levier de l'intensification du travail, comme l'indique une ouvrière française de chez Levis :

Les cadences ne sont pas imposées par la direction, chacun travaille selon ses besoins. Moi je travaille selon mes capacités, par rapport à mon budget, et à ma santé. Parce que les cadences, ça peut donner toutes sortes de maladies professionnelles, et aussi les gestes répétitifs, faut voir ce que ça peut donner au niveau des épaules. Il y a des personnes qui se sont fait opérer aux épaules par rapport à ça. Tout ça pour quoi ? Pour une performance, pour essayer d'avoir un salaire décent.

La technique change les modalités de la contrainte. L'usine d'assemblage électronique l'organise par des voies mécanisées, avec des postes assis où des femmes opèrent sur des montages qui défilent devant elles selon un rythme automatique. Tandis que la voix off d'Essere donne met en cause la fatigue visuelle et la tension nerveuse, un témoignage mentionne 18 soudures à la minute et la peur du chronométreur. Dans l'usine de Calor des années 2000, l'image montre sobrement des femmes penchées elles aussi sur des opérations de montage qu'elles paraissent effectuer dans le calme. Jusqu'à ce qu'un groupe de retraités en visite s'arrête et entame une conversation. L'évocation des cadences d'antan et d'aujourd'hui se fait dans des termes similaires, les douleurs articulaires se font écho. Taquin, un ancien demande: «Et si vous voulez arrêter, vous appuyez sur quel bouton? - Il n'y en a pas». Le summum de la sophistication vient cependant d'Indonésie où, dans une usine de confection travaillant pour Levis, l'informatisation du suivi de production permet à la contremaîtresse de suivre sur écran les arrêtstoilette de chaque ouvrière et de "repérer les mauvais travailleurs, pardon, le mauvais travail », selon les termes de la guide pour visiteurs occidentaux qui reprend immédiatement son lapsus.

À la mise en images de la dureté du travail répond la parole des femmes. De nombreux films laissent s'exprimer l'usure des travailleuses. Certains évoquent la crise de nerfs, analysée par Fanny Gallot à partir d'une scène de Coup pour coup élaborée avec des ouvrières ${ }^{9}$. Chez Grandin, d'autres ouvrières ne disent pas autre chose :

9 Gallot 2009. 
Je me rappelle une journée très, très dure. Il y a eu des crises de nerfs en chaine, à tel point que la chaîne a été arrêtée toute une journée. Ça, c'est parce qu'on exigeait d'un seul coup une production bien supérieure à celle qu'on faisait d'habitude, exactement dans les mêmes conditions de travail. Dès qu'on entre, on nous demande beaucoup, le maximum, le soir quand on rentre on est complètement vidée. Ça explique ces crises de nerfs. On n'en peut plus. C'est la fatigue nerveuse, ça vient par le bruit, et la tension avec la pièce qui arrive alors qu'on n'a pas fini celle qu'on a dans les mains.

Une ouvrière italienne fait écho :

Au bout de 8 heures, on rentre cassées à la maison. On ne sent plus nos os, et on ne se rend pas compte qu'on crève vingt ans avant.

Comme si cette fatigue allait de soi, un court-métrage letton se concentre, éberlué, sur une ouvrière qui, depuis vingt ans, casse chaque jour sans déplaisir 20000 œufs à la main, et sépare les blancs des jaunes (Egg lady). Si sa performance est exceptionnelle, elle n'est toutefois pas seule à sembler trouver une forme de calme à l'atelier (Shurale). Certains films, parfois lors de luttes, mentionnent ou soulignent l'attachement non seulement à l'emploi, mais aussi au travail, à l'activité exercée et à la vie sociale dans laquelle il s'inscrivait. La syndicaliste de l'usine française de Levis avoue en aparté, juste avant de faire assumer avec fierté la défaite par l'assemblée des ouvrières (Ouvrières du monde) :

Là, je vais quitter ma machine. Si on regarde autour de nous, ça va être vide. Pour partir où ? Ben on était bien quand même ici, oui, on était bien. Je regrette mon usine. Elle est pas à moi, mais quand même, je regrette.

\section{Les luttes et leur polysémie}

L'attrait des films sur les ouvrières vient non seulement du fait qu'elles triment, mais qu'elles se rebiffent. Près de la moitié d'entre eux évoquent des luttes qui sont l'objet essentiel de plusieurs réalisations, dont certaines sont tournées vers la mise en valeur d'héritages précieux en combinant des images d'archives et des entretiens avec d'anciennes militantes. La dureté des conditions de travail, les combats menés depuis 1945 et la construction d'un syndicalisme au féminin sont le thème conducteur du film sud-coréen $W e$ are not defeated, dont la démonstration, ample et abondante en 
images d'archives et témoignages, s'inscrit dans une logique de continuité entre passé et présent. D'autres films traduisent la redécouverte par une génération des combats menés par celle qui l'a précédée, comme ces Américains des années 1970 retrouvant les témoignages et les traces de l'action de trois pionnières du syndicalisme féminin dans le Chicago des années 1930 (Union Maids). De même, en 2009, une jeune Française d'origine portugaise mobilise de vieilles images filmiques du printemps 1974 (Nous, ouvrières de la Sogantal) pour explorer les tenants de l'occupation prolongée d'une usine textile de la banlieue de Lisbonne et interroger ses animatrices d'alors. Dans une remarquable scène de cette époque, un cercle de très jeunes ouvrières rétives écoute un homme en costume-cravate guère moins jeune, qui n'est autre que le ministre du Travail issu de la révolution des œillets, leur faire la leçon :

Les ouvriers ne peuvent pas exiger comme vous l'avez fait ici. Ils peuvent exposer leurs problèmes... Un syndicat ou un travailleur ne peut pas exiger ceci ou cela. - Il ne peut pas? - Il ne peut pas. Il peut présenter ses problèmes, demander qu'ils soient résolus ... mais pas sous forme d'exigence comme cela a été fait ici. De la façon de poser les problèmes dépendent les possibilités de trouver des solutions.

La majorité des évocations de luttes s'effectuent au présent. Certains conflits portent sur les salaires, les conditions de travail ou le despotisme de l'encadrement, mais ils sont peu nombreux à se déclencher pour ces motifs: Tout va bien (2010); We want sex equality (2011 en France) ; Maryflo (2010) ; Une part du ciel; On n'est pas des steacks hachés (2002). Les luttes sont souvent composées comme des épopées riches en péripéties. Les déclenchements de grève, les altercations, les prises de contrôle des lieux, les initiatives variées offrent dans la plupart des films de fiction des scénarios plus explicites que les documentaires. Mais ces derniers présentent parfois une crudité sidérante. Le directeur de Maryflo, qui insulte les grévistes après les avoir harcelées sur leurs machines, est d'une virulence telle que l'on croit longtemps à une farce, illusion confortée par la propension du personnage à prendre la caméra à témoin de ses frasques. Sa patronne n'est pas moins haute en couleurs, elle qui, une fois les fabrications délocalisées, déclare: «Les usines, il y a des problèmes, mais sans usine, on s'ennuie ». D'un continent à l'autre, 
les images de grévistes, de manifestants, de face à face et d'affrontements avec la police renouvellent et féminisent l'image de la lutte de classes. Dans les années 1970, cette image, même lorsqu'elle porte sur la défense de l'emploi (Lip; Grandin) demeure marquée d'une relative confiance, comme dans les films sur Lip pour lesquels les pertes d'emploi sont pour partie une toile de fond au traitement d'autres questions.

\section{Entre contestation et alternative}

Au fil du temps, l'ensemble des films concernés tend à se répartir en deux types, géographiquement distincts. En Asie, et peut-être dans d'autres continents, la thématique de contestation se poursuit dans certains films, en combinant l'emploi, les conditions de travail ou les salaires. Le documentaire au titre intrigant, Lesbian Factory suit un groupe de Philippines, sous contrat temporaire dans des entreprises taïwanaises, qui se battent pour le paiement d'arriérés de salaires. En Europe de l'Ouest, les destructions d'emploi et fermetures d'usine prennent une importance lancinante, sous forme de menace, d'état de fait ou d'empreinte d'un traumatisme passé. Le phénomène est illustré pour la France par les cas d'Epeda et de Moulinex, de Levis et de Starissima ou encore de Samsonite et de Legre-Mante, souvent liés à une délocalisation. Ses représentations diffèrent d'abord par la réalité des péripéties. Ainsi les stratégies patronales passent tantôt par de longues préparations des esprits, et tantôt par de soudaines disparitions. Selon les cas, leurs tactiques sont incarnées par des interventions dans les médias, des interviews décomplexées ou des face à face impromptus avec les grévistes. Les dynamiques de mobilisation ouvrière et les modalités d'action sont encore plus finement présentées, quand elles ne constituent pas à elles seules l'objet du film: assemblées et débats, manifestations encore, irruptions perturbatrices dans des lieux publics, débats, etc. Les différences tiennent aussi aux options cinématographiques des réalisateurs. Une partie des films perpétue l'alternance de séquences prises sur le vif et d'interviews hors de l'action, suivant le genre documentaire installé dans les années 1960-1970. D'autres s'attachent à saisir dans le cours de l'action les échanges intimistes entre ouvrières qui, insensiblement, construisent à petits pas les inflexions 
d'opinion qu'ensuite les assemblées formalisent par des interventions et des décisions collectives. Enfin, quelques films tendent à s'installer dans le temps d'après la lutte. C'est le cas d'une méditation belge dans laquelle les usines ne sont plus que des traces architecturales (Devenir). Dans l'Italie de 2011, une jeune ouvrière menacée par la fermeture de son usine réalise que son paysage familier comporte les restes d'une autre usine, bien plus grande, dont la disparition finit de s'accomplir au profit d'une spéculation touristique (In futura costruzione).

Plusieurs films offrent une alternative concrète : la coopérative. L'idée constituait déjà en 1936 la trame d'une tragi-comédie, Le crime de monsieur Lange, dans lequel Renoir traitait avec légèreté l'aventure inconséquente de midinettes. Les films sur Lip, paradoxalement, ne parlent guère de la formule pourtant attachée à ce nom. Mais le principe de fabriquer, de vendre et de se payer se trouve à la Sogantal. L'époque est évoquée, également dans un registre exclusivement féminin, avec le récit d'un groupe de femmes charpentières lassées des discriminations professionnelles et syndicales, qui créent en 1978 leur propre entreprise (Raising the roof). Plus tard, l'idée est au centre d'une fiction italienne, qui trace les péripéties industrielles, gestionnaires et affectives d'un collectif féminin formant une coopérative textile dans le Milan des années 1980 (Gentili signore). Deux autres films, une fiction puis un documentaire, s'attachent au processus qui fait passer le personnel d'une entreprise en liquidation à l'option de reprise en coopérative (L'amour sur un fil; Entre nos mains). La finesse du second tient au suivi attentif par la cinéaste des processus diffus par lesquels les ouvrières réinventent dans les années 2000 le projet. Une fois encore, celui-ci est saboté par la conjugaison patronale des banques, des concurrents et des clients de la distribution, selon la morale de classe qu'explicite la patronne de Maryflo décidant de fermer son usine : «Ils veulent tout. Qu'ils se débrouillent. Comme ça, ils iront pointer au chômage dans la dignité ».

\section{Genre au travail et travail du genre}

De façon attendue, des films montrent des discordances entre hommes et femmes à l'usine, voire entre ouvriers et ouvrières. Certaines refusent la médaille du travail que veulent leur épingler leurs 
directeurs (Revers), d'autres chantent à leur patron évaporé «Et maintenant, gros radin gros connard, mets-toi ton fric au cul jusqu'au dernier centime» (Nous, ouvrières de la Sogantal). Somme toute, ce sont les films des années 1970 qui exposent avec le plus de netteté les contradictions de genre, souvent associées avec des conflits professionnels, hiérarchiques ou de classe. Néanmoins, un film autrichien souligne un conflit interne au groupe ouvrier, lorsqu'au retour de la guerre de 1918-1918, les hommes peinant à retrouver un emploi veulent expulser des ateliers des ouvrières qui s'y sont implantées (Marianne. Ein Recht für Alle). L’hérö̈ne, épouse en même temps que déléguée syndicale, se trouve prise dans le dilemme. De façon plus ciblée, plusieurs films présentent une emprise masculine plus ou moins marquée sur les syndicats et les luttes (Rêves d'usine; 300 jours de colère). D'autres montrent combien elles sont une occasion de bousculer les rapports de genre. Le fait n'est pas nouveau. Déjà, dans le récit mémoriel des militantes américaines sur les années 1930 (Union maids), le combat contre les préjugés sexistes des ouvriers et des cadres syndicaux à l'égard des femmes qui luttaient avec eux, fit partie de la construction même du syndicalisme. Au cours des années 1970 , on voit apparaître ce combat de plus près, avec plus ou moins de virulence selon les situations et selon les films.

Des contestations de chefs syndicaux se trouvent dans certains films (Coup pour coup). Le documentaire Quand les femmes ont pris la colère (1977) retrace l'action, en Loire Atlantique, d'épouses en faveur de leur mari ouvrier, la répression contre celles-ci et les mobilisations qui s'ensuivent. Une des militantes traduit l'analyse qu'elle en fait après :

J'ai découvert qu'on a aussi de petits conflits avec les hommes, ouvriers, syndicalistes, même les militants politiques. Ils ont du mal à admettre la force que peut apporter la femme dans certaines luttes. Les femmes poussent les portes et ils ont peur, eux, de rester coincés. Ils ont besoin comme on dit d'une certaine éducation.

Sur ce point, le regard féministe offre plus d'acuité avec, en particulier, les films de Carole Roussopoulos sur Lip. Le monologue de Monique Piton est, à cet égard, une séquence d'anthologie (Lip $V$. Monique et Christine) : 
Je vais raconter un peu ce qui se passe chez Lip. Mais je vais remplacer le mot "hommes" par le mot "blancs", et le mot "femmes" par le mot "Arabes". Alors à chaque fois que je vais dire Arabes, ça veut dire des femmes.

Dans un tableau picaresque, les images du travail et celles de la conduite de la lutte entremêlent maintes formes de domination masculine, du contrôle de la parole à la formation d'une délégation au commissariat de police. L'irruption difficile des conflits de genre et du viol dans les débats d'assemblée ou la discrète évocation des violences conjugales ponctue la nouveauté des années 1968 (Lip V. Monique et Christine; Quand les femmes ont pris la colère). Enfin, les ouvrières développent des modalités d'action, chants et théâtre notamment ${ }^{10}$, que l'on trouve plus fortement représentées à propos d'usines féminines (Nous les ouvrières de la Sogantal; Entre nos mains), dont cette ancienne ouvrière portugaise indique la valeur :

Tu sais, ce sont des rimes inspirées... Ces rimes ont été faites par tout le monde. Je ne peux pas dire exactement, mais on était nombreuses et chacune y allait de son mot. La souffrance alimente notre veine poétique, ça doit être ça. Il y a beaucoup de sentiment là-dedans.

Nombre de films abordent les rapports de genre dans lesquelles les ouvrières sont prises hors du travail, suivant un schéma qu'expose en 1965 Cecilia Mangani (Essere donne) : la double journée annihile l'émancipation apportée par la vie d'usine vis-à-vis du patriarcat et de l'épuisant travail à domicile. Contre cette inégalité des rapports de couple, des films postérieurs montrent des mises en cause lors des luttes. Dans bien des cas, les femmes vont prendre « individuellement» leur décision sur les initiatives discutées à l'usine, c'est-à-dire par une discussion d'ordre familial (Entre nos mains). Néanmoins, les familles évoluent diversement (Quand les femmes ont pris la colère). Certaines se renforcent:

Ça m'a rapproché de ma femme, c'est formidable ce qu'elle a fait. (...) Je m'attendais plutôt à la réaction de la petite femme normale, la petite femme d'ouvrier, qui reste chez elle et qui dit "y en a marre, ça suffit de bouffer des pommes de terre, faut retourner travailler".

10 L'importance des chansons dans les grèves d'ouvrières a été soulignée dans Gallot 2012. 
Norma Rae offre au contraire l'archétype d'une tension croissante de l'hérö̈ne avec son mari, tandis qu'elle s'entend de mieux en mieux avec son leader syndical, en tout bien tout honneur. Dans un schéma similaire, un contremaître bienveillant peut remplacer le syndicaliste généreux face au mari brutal (Sassedkata). D'autres connaissent l'entrave du mari à l'engagement de sa femme, ou une séparation : "Après ce qui s'est passé à Tréfimétaux, ça marche plus pour nous. Mon mari gagnait pas assez d'argent, alors il est parti en déplacement». L'épouse le suit, sans réussir à éviter la rupture: «Maintenant, je suis bien, j'ai un boulot, je fais ce que je peux avec mes gosses, je me sens bien, j'ai mon petit boulot tranquille, mes enfants avec moi». Au-delà des crises et des ruptures, plusieurs films se ressemblent à suivre la solitude ordinaire d'ouvrières, employées à Moulinex comme dans un abattoir russe, le vignoble bordelais, une pâtisserie lettonne ou une imprimerie autrichienne (Mon travail c'est capital; Femmes précaires; Egg lady; Elefantenhaut). Tantôt l'héroïne ouvrière a ses enfants à sa seule charge (Laisse un peu d'amour), tantôt sa mère âgée ou son vieux père. La difficulté de trouver un compagnon vivable peut n'être jamais évoquée ou, au contraire, fournir la trame d'une quête opiniâtre propice à bien des facéties (With love, Lily). Dernier mode de vie présenté, les pensionnats pour jeunes ouvrières peuvent apparaître comme des rémanences d'autrefois ( $U n$ sac de puces). Il est vivace dans la nouvelle industrie du Vietnam ou de Taïwan. La réalisatrice du film taïwanais Lesbian factory, tombe au cours d'une lutte sur la propension de jeunes immigrées entassées dans des dortoirs sommaires à trouver un réconfort dans une homosexualité qui, parfois, s'évanouit lorsque les fins de contrat dissolvent l'effectif.

\section{Tête basse, tête haute}

S'insurgeant contre une présentation larmoyante faite par une chaîne de télévision des ouvriers de Samsonite, la réalisatrice de Liquidation totale explique avoir cherché à souligner leur combativité, leur résistance, leur courage. Plus que la réalité du film, sobre en la matière, l'argument renvoie à la veine héroïque des films produits dans les années 1970 et qu'on retrouve dans des productions plus récentes, masculines et 
féminines (300 jours de colère; Garder la tête haute). Sans toujours viser ce terrain-là, la plupart des cinéastes s'entendent pour mettre en relief la dignité des ouvrières représentées et cherchent une bienveillance sans condescendance, qu'ils réalisent par différentes options concrètes ${ }^{11}$. Pour les dernières décennies, les réponses se trouvent souvent dans un cinéma d'accompagnement, plutôt que d'une posture de porte-parole. C'est ainsi que, lorsque la réalisatrice vietnamienne du film Rêves d'ouvrières, munie d'une caméra légère, interroge dans un dortoir de Hanoï une ouvrière sur sa condition, celle-ci répond de laisser là la caméra et de venir s'asseoir à côté d'elle pour qu'elle puisse « lui parler comme à une sœur». En s'exécutant, la réalisatrice accepte d'adopter une relation de proximité et d'égalité.

L'âge est un indicateur significatif de la difficulté d'être ouvrière. La plupart des personnages jeunes cherchent à tout prix à éviter l'usine et la vie d'ouvrière, et peuvent prendre tous les risques pour y parvenir (Sur la planche; La vie rêvée des anges). Dans En avoir (ou pas), l'héroïne jette à son compagnon, terrassier, le refus paradoxal de continuer à le voir : «De toute façon, vous êtes pas fait pour moi. Trop paumé, ouvrier. Vous êtes miteux. On dirait moi ». Elle a, pour fuir son destin, quitté la conserverie de poisson de Boulogne-sur-Mer dont elle croit longtemps garder l'odeur sur les mains et traversé la France. L'évasion est dans certains cas traitée sous l'angle du loisir-passion, où s'investissent tous les espoirs (Secret society). L'acceptation, elle, ne se fait pas sans peine comme le montre le dialogue entre réalisatrice et ouvrière (Calor, une usine en perspective : Rovetta, le dragon et la piewvre) :

Je vous ai demandé quel métier vous faisiez chez Calor, et vous m'avez reprise en me disant : «Ah, le travail ». Vous faites une différence ? - Un métier, c'est une qualification. Moi, tout ce que j'ai fait, c'est du répétitif. C'est pas un métier. - Vous faisiez allusion à votre fille. Quand on doit marquer la profession des parents, et qu'elle vous demandait, vous disiez quoi ? - Je me disais ouvrière devant les autres parents d'élèves. J'aimais pas trop. Je ne devrais pas. J'ai pas de honte à avoir d'être ouvrière. C'est honorable, je ne fais pas quelque chose de malhonnête. Quand les petites filles disent ma maman est institutrice, secrétaire... C'est tout bête, mais... Pourtant, je n'ai pas honte de faire ça. C'est honnête, c'est

11 Leur préoccupation se rapproche dans bien des cas de Claude Grignon et JeanClaude Passeron, Le Savant et le Populaire. Misérabilisme et populisme en sociologie et en littérature, Gallimard/Le Seuil, 1989. 
gratifiant, je touche mon salaire, Mais c'est quelquefois le regard des autres. Selon avec qui vous êtes : ah bon, vous travaillez en usine. Mon mari me dit que je ne dois pas être comme ça. Mais moi, je le ressens.

Cette ouvrière dit combien, dans son enfance, elle était fière du métier - vrai celui-là - de sa mère couturière. Comme en écho, une Flamande de chez Levis dit avoir mis ses plus beaux habits pour son premier jour d'usine (Ouvrières $d u$ monde). Faut-il y voir la fin d'une dignité ouvrière forte jusqu'aux débuts de la désindustrialisation et de l'effritement du mouvement ouvrier? Un autre témoignage, d'une militante communiste de 1976, incite à ne pas réinterpréter ce passé (Quand les femmes ont pris la colère):

Moi, je lutte pour être heureuse. Pour être une femme, bien dans sa peau. Pas fière d'elle, mais... La chose que je voudrais le plus, ce serait d'avoir un travail qui me plait. De ne pas avoir honte en disant «je suis manutentionnaire». Qui va nous l'apporter, ce truc-là, ce serait un changement politique. Et je pense qu'après, les rapports entre les hommes et les femmes, ça serait transformé. Ça sera jamais parfait, on va vers la perfection, sans jamais l'atteindre, mais...

Dans une définition où, là aussi, se combinent l'intime et le public, le travail et ce qui lui est extérieur, c'est encore à une autre temporalité, le futur en l'occurrence, que renvoie cette quête de dignité, alors que le présent n'est synonyme que d'insatisfaction.

\section{Filmographie}

1936 : Le Crime de monsieur Lange, Jean Renoir, France (đ). 1962 : Un sac de puces, Vera Chytilová, Tchécoslovaquie. 1965 : Essere donne, Cecilia Mangini, Italie. 1973 : Lip 1. Monique (Lip), Carole Roussopolos, France. 1972 : Coup pour coup, Marin Karmitz, France (ठ̋). Tout va bien, Jean-Luc Godard, France $(\hat{O}) .1974$ : Humain trop humain, Louis Malle, France (ठ). 1975 : Grandin, Nat Lilenstein, France. 1976 : Quand les femmes ont pris la colère, Soisig Chappedelaine et René Vauthier, France; Lip V. Monique et Christine, Carole Roussopolos, France ; Union Maids, Julia Reichert, James Klein, Miles Mogulescu, USA. 1979: Norma Rae, Martin Ritt, USA (ô). 1982: Laila, Diane Beaudry, Canada. 1984: Marianne. Ein recht für Alle, Käthe Kratz, Autriche. 1987: Naplo Szerelmeimnek, Marta Meszaros, Hongrie. 1988: Komplizinnen, Margit 
Czenki, RFA. 1989: Gentili signore, Adriana Monti, Italie. 1990 : Sassedkata (La voisine), Adela Peeva, Bulgarie. 1991 : Shurale, Renita et Hannes Lintorp, URSS/Estonie. 1992 : Revers, Daisy Lamothe, France. 1995: En avoir (ou pas), Laetitia Masson, France. 1997 : Laisse un peu d'amour, Zaïda Ghorab-Volta, France; Rue des filles de Chantelle, Danielle Lefebvre et Dominique Ménard, France; Maryflo (série Stripease), Olivier Lamour, France (ठึ). 1998: La vie rêvée des anges, Erick Zonca, France (ठ઼). 2000 : Mon travail, c'est capital, Marie-Pierre Bretas, Raphaël Girardot et Laurent Salters, France ; Les filles de la sardine, Marie Hélia, France. Daughter of the sun, Maryam Shahriar, Iran; Egg Lady, Una Celma, Lettonie; Owrières du monde, Marie-France Collard, Belgique. L'amour sur un fil, Michaela Watteaux, France. 2001: Paroles de Bibs, Jocelyne Lemaire-Darnaud, France ; Une part du ciel, Bénédicte Liénard, Belgique; Secret society, Imogen Kimmel, Allemagne ; Rêves d'usine, Luc Decaster, France (ठ). 2002 : On n'est pas des steacks hachés, Anne Galland, Alina Arouali, France; Sauf la lutte, Catherine Tréfousse, France; Manjuben, Truck driver, Sherna Dastur, Inde ; Owvrier, c'est pas la classe, Patrick Jan, France (đ). 300 jours de colère, Marcel Trillat, France (ठđ). 2003 : With love, Lilly, Larisa Sadilova, Russie. 2005 : Calor, une usine en perspective: Rovetta, le dragon et la pieuvre, Martine Arnaud-Goddet, France ; Raising the Roof, Veronica Selver et Françoise Flamant, USA ; Femmes précaires, Marcel Trillat, France $\left({ }^{\top}\right) .2006$ : Rêves d'ouvrières, Tran Phuong Thao, Vietnam ; We are not defeated, Lee Hye-Ran, Corée. 2009 : Liquidation totale, Hélène Desplanques, France; Elefantenhaut, Ulrike Putzer, Severin Fiala, Autriche. 2010 : We want sex equality, Nigel Cole, Royaume-Uni (ठ). Sochaux, cadences en chaîne, Laurence Jourdan, France ; Entre nos mains, Mariana Otero, France; Lesbian factory, Susan Chen, Taiwan; Nous, ourrières de la Sogantal, Nadejda Tilhou, Portugal. 2011 : Disparaissez, les ouvriers, Christine Thépenier et Jean-François Priester, France; Devenir, Loredana Bianconi, Belgique; In futura costruzione, Giulia Casagrande, Italie; Sur la planche, Leila Kilani, Maroc, Allemagne, France. Garder la tête haute, Martine Gonthié, France.

\section{Bibliographie}

Beaud Stéphane \& Michel Pialoux, 1999, Retour sur la condition ouvrière. Enquête aux usines de Pengeot-Sochaux, Paris, Fayard. 
CADÉ Michel, 2004, L'Écran bleu. La représentation des ouvriers dans le cinéma français, Perpignan, Presses universitaires de Perpignan.

GALLOT Fanny, 2009, « La "crise de nerfs", de la souffrance à la résistance ? ", CLIO, Histoire, Femmes et Sociétés, 29, « 68, révolutions dans le genre ? », p. 153-164.

—, 2012, Les Ouvrières, des années 68 au très contemporain : pratiques et représentations, thèse de doctorat en histoire, université Lumière Lyon 2, sous la dir. de Michelle Zancarini-Fournel.

Hatzfeld Nicolas, Rot Gwenaële \& Alain Michel, 2006, «Le travail au cinéma. Un réapprentissage de la réalité sociale », Esprit, 326, p. 78-99.

LiNDEPERG Sylvie, 2007, Nuit et brouillard : un film dans l'bistoire, Paris, Odile Jacob.

Rot Gwenaelle \& Laure DE VERDALLE (dir.), 2013, Le Cinéma : travail et organisation, Paris, La Dispute.

VIGNA Xavier, 2012, Histoire des ouvriers en France au XX'e siècle, Paris, Perrin. 